\title{
Integrated Pharmaceutical Logistics System Implementation in Chagni Primary Hospital and Injibara General Hospital, Awi Zone, Ethiopia
}

\author{
Bereket Bahiru Tefera $\mathbb{D D}^{\prime}$ \\ Wubetu Yihunie (iD) \\ Azmeraw Bekele iD ${ }^{2}$ \\ 'Department of Pharmacy, College of \\ Health Science, Debre Markos University, \\ Debre Markos, Amhara, Ethiopia; \\ ${ }^{2}$ Institute of Health Science, Jimma \\ University, Jimma, Oromia, Ethiopia
}

\begin{abstract}
Background: Integrated Pharmaceutical Logistics System is the primary mechanism through which all public health facilities in Ethiopia get essential pharmaceutical products from their main supplier. Pharmaceuticals should be managed appropriately because they are part of the link between the patient and health services and account for up to half of the healthcare budget. This study aimed to assess the status of Integrated Pharmaceutical Logistics System implementation at both Chagni Primary Hospital and Injibara General Hospital.
\end{abstract}

Methods: Facility-based descriptive study design supported with a qualitative study design was used. Face-to-face interviews, observation of practices, and document review were conducted to gather quantitative data. Besides, the qualitative data were collected through indepth face-to-face interviews. Frequency and percentage were computed, and the results were briefly described in text and displayed in tables and graphs. The qualitative data were transcribed manually, and thematic analysis was done.

Results: All IPLS materials were available in both hospitals, but the stock recording card was not available at Injibara General Hospital. About $90 \%$ and $100 \%$ of the dispensing units in Chagni Primary Hospital and Injibara general hospital were utilizing bin-cards, respectively. Besides, $50 \%$ and $80 \%$ of the bin-cards in Chagrin Primary Hospital and Injibara General Hospital were regularly updated, respectively. About $80 \%$ and $75 \%$ of the IFRRs in Chagni Primary Hospital and Injibara General Hospital reported valid data, respectively. Besides, $66.67 \%$ and $50 \%$ of the RRFs reviewed at Chagni Primary Hospital and Injibara General Hospital reported valid data, respectively. Medicine stockouts, poor staff commitment, and workload were the major bottlenecks for IPLS execution.

Conclusion: The status of most of the IPLS implementation indicators in both hospitals was good. Especially, the availabilities of IPLS materials and the calculation accuracy of both RRFs and IFRRs were encouraging in both hospitals. However, the validity of the data reported in IFRRs and RRFs, and the status of the storage conditions in both hospitals need some improvement during the implementations of IPLS.

Keywords: IPLS implementation, report and requisition form, bin-card, hospital

\section{Introduction}

The World Health Organization (WHO) estimates that around one-third of the world's population lack access to essential medicines and even this amount increases in the poorest parts of Africa and Asia. ${ }^{1}$ The causes of poor access and availability of medicines were multifaceted and some of the contributing factors for these complications are irrational use of medicines, unsustainable financing
Correspondence: Bereket Bahiru Tefera Department of Pharmacy, College of Health Science, Debre Markos University, Debre Markos, Amhara, Ethiopia

Tel +25I90988I252

Email brktbahiru@gmail.com 
mechanisms, and ineffective health care supply chain systems to deliver medicines to the final consumers. ${ }^{1,2}$ Besides, in the pharmaceutical industry, there is a growing concern with supply chain sustainability during the COVID-19 pandemic. $^{3}$

The delivery of health care services needs the availability of safe, effective, and affordable pharmaceuticals and related medical supplies of the required quality, with adequate quantity at all times. ${ }^{1}$ These pharmaceuticals should be managed appropriately because usually, they account for up to half of the health care budget, and medicines are part of the link between the patient and health services. ${ }^{4}$ Because even minor disruptions in the pharmaceutical supply chain can result in severe disasters, pharmaceutical product distribution must combine cost minimization with strict adherence to service standards while accounting for risks due to uncertainty. ${ }^{5}$ When hospitals faced drug shortages, they had to postpone treatments and rely on less effective and more expensive substitutes. ${ }^{6}$

The Federal Minister of Health (FMoH) of Ethiopia has been working to ensure that all Ethiopians have a successful and high-performing supply chain of healthcare that guarantees equal access to quality drugs and related supplies. Although substantial progress has been made, several problems remain, including an insufficient supply of quality and affordable essential pharmaceuticals, poor storage conditions, and inadequate inventory management. ${ }^{7}$ To encourage the public sector healthcare supply chain, the FMoH introduced a comprehensive supply chain strategic planning process that emphasizes the integration of all health commodities into one supply chain, which was the Integrated Pharmaceutical Logistics System (IPLS). IPLS began in 2009 and the mandate for implementing it was given to Pharmaceutical Fund and Supply Agency (PFSA). ${ }^{8}$

IPLS is a system used as a pharmaceutical reporting and distribution system that integrates the management of HIV/AIDS, malaria, TB and leprosy, EPI, MCH, and other purchased essential drugs. It aims to ensure that the six rights of pharmaceutical supply chain management are fulfilled by ensuring the right products in the right quantity, of the right quality, at the right place, at the right time, and for the right cost. ${ }^{1,9}$ The IPLS is the primary mechanism through which all public health facilities obtain essential medicines. 9 The IPLS has three main components including policies and guidelines for logistics management information system (LMIS), inventory control, and storage of pharmaceuticals at all levels of the public health supply chain system throughout the country. ${ }^{10}$ Besides, each component has its own set of indicators for measuring improvements and performances of the system. ${ }^{10,11}$

Different reports from various parts of the country show that the implementation of IPLS has improved the recording and reporting of LMIS, storage practices, as well as the availability of essential pharmaceuticals at service delivery points. ${ }^{10}$ However, there is no available study conducted to assess the IPLS implementation in both Chagni Primary Hospital and Injibara General Hospital and even in the region where those hospitals were found. Therefore, this study aimed to assess the status of IPLS implementation in these hospitals, which are the main hospitals providing healthcare services for the majority of the population in that region.

\section{Methodology \\ Study Setting and Period}

The study was conducted to assess the status of IPLS implementation in Chagni Primary Hospital and Injibara General Hospital, which are found in Awi zone, Amhara regional state, Ethiopia. Injibara General Hospital is located in northwest Ethiopia, which is 144 and 450 kilometres far from Bahir Dar (which is the capital city of Amhara Regional State), and Addis Ababa, respectively. Besides, it was serving an estimated 1.2 million population in Awi zonal administration. ${ }^{12}$ Chagni Primary Hospital is located in the Awi Zone of the Amhara Regional state, and it is 165 kilometres from Bahir Dar and 502 kilometres far from Addis Ababa.

\section{Study Design}

A facility-based descriptive study design supported with a qualitative study was employed for assessing the status of IPLS implementation.

\section{Source Population}

The source populations of this survey were Chagni Primer Hospital and Injibara General Hospital, the IPLS-related documents, and the healthcare professionals of these hospitals.

\section{Study Populations}

The study units of this study were dispensing units, IPLSrelated documents in selected dispensing units (bin-cars, 
IFRR, and RRF), store manager, and pharmacy head of each hospital.

\section{Sample Size Determination and Sampling Procedure}

The data regarding the status of bin-card management were collected through reviewing the last 2 months (before data collection) bin-cars of 3 randomly selected pharmaceutical items that were managed by each selected dispensing unit to assess bin-card management (the selected dispensing units were the pharmaceutical store, outpatient pharmacy, inpatient pharmacy, antiretroviral (ART) pharmacy, and emergency pharmacy, MCH clinic, TB clinic, Medical laboratory, X-ray, and OR department). These pharmaceutical items in each dispensing unit were randomly selected during the data collection period, from the shelf where all pharmaceuticals were arranged. Therefore, a total of 30 bin-cars were reviewed.

Besides, the data required for the Internal Facility report and Re-Supply Form (IFRR)-related indicators were gathered from the last 4 IFRR documents (ie, prior to the data collection time) reported by 5 purposively selected dispensing units (DUs), such as outpatient pharmacy, inpatient pharmacy, antiretroviral (ART) pharmacy, emergency pharmacy, and tuberculosis (TB) clinic. These dispensing units were selected based on the perceived high patient flow rate relative to the other dispensing units. Therefore, a total of 20 IFRR documents were reviewed. Moreover, the data of 3 randomly nominated pharmaceutical items reported by each selected IFRR were reviewed. To collect data regarding the Report and Requisition Form (RRF)-related indicators, the RRF documents of the last 1 year (which means 6 RRF from each hospital) were reviewed.

The pharmaceutical store managers, the pharmaceutical supply chain officer, and the pharmacy head of each hospital were selected as key informants for collecting the qualitative data regarding the challenges of IPLS implementation in Chagni Primary Hospital and Injibara General Hospital. Therefore, a total of 6 key informants (1 store manager, 1 pharmaceutical supply chain officer, and 1 pharmacy head from each hospital) were interviewed.

\section{Data Collection Tool and Procedures}

Structured and semi-structured questionnaires in the English language were prepared based on the Logistics
Indicators Assessment Tool (LIAT). ${ }^{13}$ The structured questionnaire had two sections. The first section had questions to assess the socio-demographic characteristics of the selected hospitals and hospital pharmacy staff (who are primarily responsible for the majority of the IPLS implementation). The second section included questions important to capture the data required to compute the selected IPLS implementation indicators, which were used to measure the status of IPLS implementation. Face-to-face interviews with store managers and pharmacy heads, document review, and physical observations of the practices were conducted to gather quantitative data. Besides, a semistructured questionnaire was used to collect qualitative data regarding the challenges of IPLS implementation through face-to-face interviews with key informants.

\section{Data Processing and Analysis}

Once the questionnaires were filled, the principal investigators evaluated their completeness and the quantitative data was entered into a Microsoft Excel spreadsheet. After the appropriate data cleaning process is done, Microsoft Excel version 16 was used to calculate all indicators used to evaluate the status of IPLS implementation in both hospitals. Descriptive statistics (frequency and percentage) were computed, and the results were briefly described with texts and displayed using tables and graphs. The data from each hospital were analyzed and presented separately. The qualitative data gathered through face-to-face interviews were transcribed manually, and thematic analysis was done.

\section{Measurements of IPLS Implementation Status \\ Bin-Card Management-Related Indicators}

1. Percentage of dispensing units utilizing bin-cards: it was calculated as the number of dispensing units utilizing bin-card divided by the total number of dispensing units assessed (ie, 100 dispensing units) and multiplied by 100. Dispensing units that utilized bin-card, during the last 2 months (ie, prior to the data collection period), for all 3 randomly selected items arranged on their shelf were considered as "Utilizing Bin-card".

2. Percentage of dispensing units regularly updated their bin-cards: it was calculated as the number of dispensing units regularly updated their bin-cars divided by the total number of dispensing units 
assessed (ie, 5 bin-cars) and multiplied by 100 . If the bin-cars of all 3 randomly selected pharmaceutical items were regularly updated during the last 2 months (ie, prior to the data collection period), the dispensing units were considered as "updated".

\section{IFFR-Related Indicators}

1. Percentage of IFRR submitted on time: it was calculated as the number of IFRR submitted on time divided by the number of IFRR assessed and times 100. The IFRRs were considered as "submitted on time" when the date wrote on their IFRR is consistent with the schedule stated by the store manager.

2. Percentage of IFRR with valid data: it was calculated as the number of IFRR with valid data divided by the total number of IFRR assessed and times by 100. The IFRR was considered as "valid" when all data of 5 randomly selected items that are reported in the IFRR were accurately transferred from data sources (ie, bin-card) to IFRR.

3. Percentage of IFRR with Accurate data: it was calculated as the number of IFRR with accurate calculation divided by the total number of IFRR assessed and times by 100 . The IFRR was considered "accurate" when all calculations of 5 randomly selected items that are reported in the IFRR are correct.

\section{RRF-Related Indicators}

1. Percentage of RRF submitted on-time: it was calculated as the number of RRF submitted on-time divided by the number of RRF submitted to EPSA times 100. According to IPLS, RRF submitted before the 10th day of the reporting month was considered as "submitted on-time".

2. Percentage of RRF reported valid data: it was calculated as the number of RRF with valid data divided by the total number of RRF assessed and times by 100 . RRF was considered as "valid" when all data of 10 randomly selected items that are reported in the RRF were accurately transferred from data sources (ie, bin-card) to RRF.

3. Percentage of RRF with the accurate calculation: it was calculated as the number of RRF with accurate calculation divided by the total number of RRF assessed and times by 100 . The RRF was considered as "accurate" when all calculations of 10 randomly selected items that are reported in the RRF are correct.

\section{Storage Practices-Related Indicator}

1. Percentage of storage conditions that comply with the standards: it measures the percentage of storage conditions that comply with the standard storage guidelines recommended by IPLS. It was calculated as the number of storage conditions fulfilled divided by the number of storage conditions assessed.

According to the National Research Ethical Review Guideline of Ethiopia, when the purpose of the study is to examine government programs designed to provide the public good or service and when information has been collected by the investigator in such a way that it is impossible to identify study participants, it is exempted from ethical review. ${ }^{12-14}$ Before starting data collection, verbal permission for data collection was granted by each public health facility. Verbal consent was obtained from all respondents and confidentiality of the information was assured to them. Since the study involves no more than minimal risk to participants, verbal informed consent is acceptable under the National Research Ethical Review Guideline. $^{14}$

\section{Result}

\section{Characteristics of the Hospitals and Professional's Profile}

Both hospitals were implementing both paper-based and Health Commodities Management Information System (HCMIS)-based IPLS for managing the supply chain of health commodities. Besides, almost all of the pharmacy personnel working in Chagni Primary Hospital, 15 (93.75), were IPLS-Untrained. Half of the pharmacy personnel working in Injibara General Hospital, 7(50\%), were IPLStrained (Table 1).

\section{Availability of IPLS Materials at Each Hospital During the Date of Visit}

All the IPLS materials were available in Chagni Primary Hospital and apart from IFRR and RRF (which were prepared by the hospital itself), most of the materials were donated by Ethiopian Pharmaceutical Supply Agency (EPSA). Additionally, except for the Stock Record Card, all other IPLS materials were available in Injibara General Hospital. And the source of most materials was the Ethiopian Pharmaceutical Supply Agency (Table 2). 
Table I The Characteristics of the Hospitals and Professional's Profile

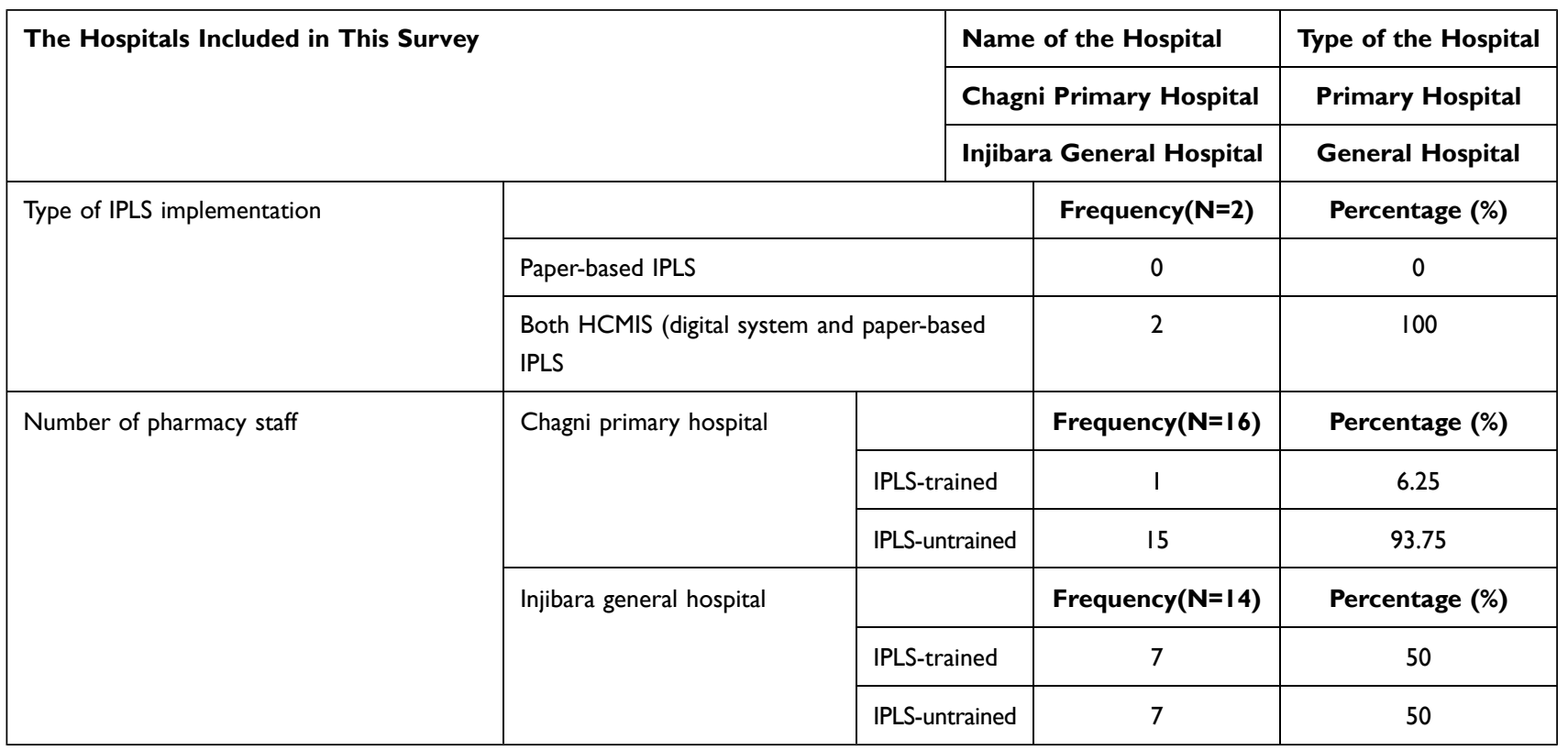

Table 2 Availability of IPLS Materials at Each Hospital During the Date of Visit

\begin{tabular}{|l|l|l|l|l|}
\hline \multirow{2}{*}{} & \multicolumn{2}{|l|}{ Chagni Primary Hospital } & \multicolumn{2}{l|}{ Injibara General Hospital } \\
\cline { 2 - 5 } & Available & Source of the Material & Available & Source of the Material \\
\hline Bin Cards & Yes & Donated by EPSA & Yes & Donated by EPSA \\
\hline Stock Record Cards & Yes & Donated by EPSA & No & Not-available \\
\hline Internal Facility Report-Re-supply (IFRR) & Yes & Prepared by the hospital & Yes & Prepared by the hospital \\
\hline Facility Report and Re-supply (RRF) & Yes & Prepared by the hospital & Yes & Prepared by the hospital \\
\hline IPLS SOP & Yes & Donated by EPSA & Yes & Donated by EPSA \\
\hline Job Aids & Yes & Donated by EPSA & Yes & Donated by EPSA \\
\hline
\end{tabular}

\section{Bin-Card Management-Related Indicators}

The majority of dispensing units in Chagni Primary Hospital, 9(90\%), were utilizing bin-cars. Additionally, all dispensing units in Injibara General Hospital, 10 $(100 \%)$, were utilizing bin-cars. Among all dispensing units assessed by this review in Chagni Primary Hospital, $5(50 \%)$ of them were not updating their bin-cards. Besides, $8(80 \%)$ of the dispensing units assessed by this survey in Injibara General Hospital were updating bincards (Figure 1).

\section{IFRR-Related Indicators}

Among all the IFRR reviewed in Chagni Primary Hospital, $11(55 \%)$ of them were submitted on time. Likewise, 12
$(60 \%)$ of the IFRR reviewed in Injibara General Hospital were submitted according to the schedule.

Among all the IFRRs assessed in Chagni Primary Hospital, $16(80 \%)$ of them reported valid data. Besides, among specific data assessed within IFRRs of Chagni Primary Hospital, all IFRRs, 20 (100\%), reported valid data of the "beginning balance". On the contrary, 17 (85\%) IFRRs reported the valid "ending balance" data, which is the least valid data reported by IFRRs. Additionally, among all the IFRRs in Injibara General Hospital, 15 (75\%) of them reported valid data. In detail, the data of "beginning balance" were valid in all IFRRs reviewed. However, the "ending balance" was the least data reported by IFRRs. It was valid only in the $16(80 \%)$ of the swotted IFRRs (Table 3$)$. 
Bin-card management related indicators

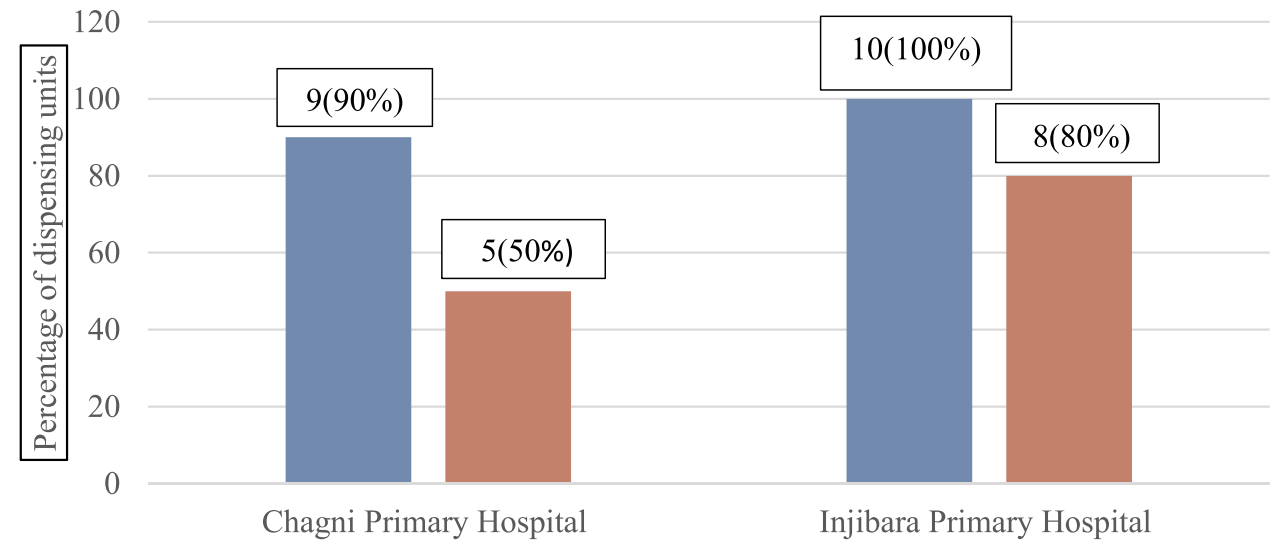

- Dispensing units utilizing Bin-cards $\square$ Dispensing units regularly updated their Bin-cards

Figure I The descriptive statistics of bin-card management-related indicators.

The overall number of IFRRs in Chagni Primary Hospital with accurate calculations was 17 (85\%). According to this survey, both the calculations of "Calculated Consumption" and the "Maximum Stock Quantity" in 19 (95\%) IFRRs reviewed in Chagni primary hospital were \%accurate. Likewise, "Quantity need to reach Maximum" calculations of 17 (85\%) IFRRs were accurate. Among all IFRRs assessed in Injibara General Hospital, $18(90 \%)$ of them had accurate calculations.
Specifically, the calculations of both the "Calculated Consumption", and "Quantity need to reach maximum" were accurate in 19 (95\%) of IFRRs examined with this survey. And, the calculations of the "Maximum Stock Quantity" were accurate in 18 (90\%) of IFRRs examined.

\section{RRF-Related Indicators}

In both Chagni Primary Hospital and Injibara General Hospital, $6(100 \%)$ of RRFs reviewed in each hospital,

Table 3 The Descriptive Statistics of IFRR-Related Indicators

\begin{tabular}{|c|c|c|c|c|c|}
\hline \multirow[t]{2}{*}{ Indicators } & & \multicolumn{2}{|c|}{ Chagni Primary Hospital } & \multicolumn{2}{|c|}{ Injibara General Hospital } \\
\hline & & $\begin{array}{l}\text { Frequency } \\
(\mathrm{N}=\mathbf{2 0})\end{array}$ & $\begin{array}{l}\text { Percentage } \\
\text { (\%) }\end{array}$ & $\begin{array}{l}\text { Frequency } \\
(n=20)\end{array}$ & $\begin{array}{l}\text { Percentage } \\
\text { (\%) }\end{array}$ \\
\hline $\begin{array}{l}\text { IFRR submitted on } \\
\text { time }\end{array}$ & The date on the IFRR consistent with its schedule & 11 & 55 & 12 & 60 \\
\hline \multirow{5}{*}{$\begin{array}{l}\text { IFRR reported valid } \\
\text { data }\end{array}$} & "Ending balance" on IFRR versus on the bin-card & 17 & 85 & 16 & 80 \\
\hline & $\begin{array}{l}\text { "Beginning balance" of the IFRR to the "Ending balance" in } \\
\text { the previous IFRR }\end{array}$ & 20 & 100 & 20 & 100 \\
\hline & $\begin{array}{l}\text { "Quantity Received" on the current IFRR with the } \\
\text { "Quantity Supplied" of previous IFRR }\end{array}$ & 18 & 90 & 19 & 95 \\
\hline & $\begin{array}{l}\text { "Calculated Consumption" versus the sum of "Quantity } \\
\text { Issued" of the bin card }\end{array}$ & 19 & 95 & 19 & 95 \\
\hline & Overall IFRR reported valid data & 16 & 80 & 15 & 75 \\
\hline \multirow{4}{*}{$\begin{array}{l}\text { IFRR with accurate } \\
\text { calculations }\end{array}$} & "Calculated Consumption" & 19 & 95 & 19 & 95 \\
\hline & "Maximum Stock Quantity” & 19 & 95 & 18 & 90 \\
\hline & "Quantity need to reach maximum" & 17 & 85 & 19 & 95 \\
\hline & Overall IFRR with accurate calculation & 17 & 85 & 18 & 90 \\
\hline
\end{tabular}


Table 4 The Descriptive Statistics of RRF-Related Indicators

\begin{tabular}{|c|c|c|c|c|c|}
\hline \multicolumn{2}{|l|}{ Indicators } & \multicolumn{2}{|c|}{ Chagni Primary Hospital } & \multicolumn{2}{|c|}{ Injibara General Hospital } \\
\hline & & \multirow{2}{*}{$\begin{array}{l}\begin{array}{l}\text { Frequency } \\
(\mathrm{N}=6)\end{array} \\
6\end{array}$} & \multirow{2}{*}{$\begin{array}{l}\begin{array}{l}\text { Percentage } \\
(\%)\end{array} \\
100\end{array}$} & \multirow{2}{*}{$\begin{array}{l}\begin{array}{l}\text { Frequency } \\
(N=6)\end{array} \\
6\end{array}$} & \multirow{2}{*}{$\begin{array}{l}\text { Percentage } \\
\text { (\%) } \\
100\end{array}$} \\
\hline RRF submitted on time & $\begin{array}{l}\text { The date on the RRF is consistent with the } \\
\text { schedule }\end{array}$ & & & & \\
\hline \multirow{4}{*}{$\begin{array}{l}\text { RRF with accurate } \\
\text { calculation }\end{array}$} & "Calculated Consumption" is accurate & 6 & 100 & 6 & 100 \\
\hline & "Maximum Stock Quantity” is accurate & 6 & 100 & 6 & 100 \\
\hline & "Quantity need to reach maximum" is accurate & 6 & 100 & 6 & 100 \\
\hline & $\begin{array}{l}\text { Overall percentage of RRF with accurate } \\
\text { calculation }\end{array}$ & 6 & 100 & 6 & 100 \\
\hline \multirow[t]{6}{*}{ RRF with valid data } & Ending balance & 4 & 66.67 & 6 & 100 \\
\hline & Beginning balance & 6 & 100 & 6 & 100 \\
\hline & Quantity Received & 6 & 100 & 5 & 83.33 \\
\hline & Calculated Consumption & 6 & 100 & 6 & 100 \\
\hline & Days out of stock & 5 & 83.33 & 3 & 50 \\
\hline & Overall percentage of RRF with valid data & 4 & 66.67 & 3 & 50 \\
\hline \multirow{2}{*}{\multicolumn{2}{|c|}{ Percentage of storage conditions comply with the standards }} & $\begin{array}{l}\text { Frequency } \\
(\mathrm{N}=14)\end{array}$ & $\begin{array}{l}\text { Percentage } \\
\text { (\%) }\end{array}$ & $\begin{array}{l}\text { Frequency } \\
(\mathrm{N}=14)\end{array}$ & $\begin{array}{l}\text { Percentage } \\
\text { (\%) }\end{array}$ \\
\hline & & 12 & 85.71 & 10 & 71.42 \\
\hline
\end{tabular}

were submitted on time to Ethiopian Pharmaceutical Supply Agency (EPSA). Besides, among all RRFs reviewed in this study, the overall percentage of RRFs with accurate calculation reported in both hospitals was $100 \%$.

From all RRFs evaluated in Chagni Primary Hospital, 4(66.67\%) of them reported valid data. Among the specific data reported on RRFs, the "ending balance" and "days out of stock" were the most frequently invalid data that were valid only in 4(66.7\%), and 5(83.33\%) of all RRFs reviewed in Chagni Primary Hospital. Besides, only $3(50 \%)$ of the RRFs evaluated in the Injibara General Hospital reported valid data. Among the specific data reported on RRFs, "days out of stock" was the most frequently reported invalid data, and it was valid only in $50 \%$ of the RRFs evaluated by this study. Besides, the "quantity received" was the next frequently reported invalid data, and it was valid in 5 $(83.33 \%)$ of the RRFs reviewed in Injibara General Hospital (Table 4).

\section{Storage Condition-Related Indicator}

This study also assessed the storage conditions in pharmaceutical stores of Chagni Primary Hospitals and Injibara General Hospitals. Among 14 storage conditions assessed in Chagni Primary Hospital, 12 (85.71\%) of them comply with the standard storage conditions recommended by IPLS. Besides, from 14 storage conditions evaluated in Injibara General Hospital, $10(71.43 \%)$ of them met the standard medicine storage conditions recommended by IPLS (Table 4).

\section{Qualitative Study}

In-depth face-to-face interviews were done with key informants to identify challenges related to IPLS implementation in their respective hospitals and all of them emphasized that frequent pharmaceutical products stockout at EPSA (besides, at private wholesalers) was the major challenge that distorting the IPLS implementation. As one of the key informants of Injibara General Hospital said that; 
... currently product availability in this country is very poor. Even the availability at our primary supplier, which is EPSA, is very low because they cannot procure due to the national dollar currency shortage ....

Besides, the other challenge that all key informants mentioned was the attitude of the staff engaged with IPLS implementation. As one of the respondents from Chagni Primary Hospital said that;

... even though we always try to discuss with them, most of the staff responsible to carry out IPLS activities believe that IPLS is their optional responsibility ....

The other challenge mentioned by most of the respondents was the limited number of pharmacy staff in their hospitals. As one of the respondents from Injibara General Hospital said that;

... the number of pharmacy personnel in our hospital is much lower than the required staff. Due to this, the workload is so high for pharmacists and patients have to wait for a long time before they get their medicines ....

\section{Discussion}

This study assessed the IPLS implementation using bin-card management-related indicators, IFRR-related indicators, and RRF-related indicators, and storage conditions-related indicators.

The availabilities of IPLS materials (Bin-cards, Stock Record Cards, IFRR, RRF, IPLS SOP, and Job Aids) were reported $100 \%$ in the Chagni Primary Hospital. These results are consistent with the national IPLS survey conducted by PFSA $^{7}$ and another study done in the Jimma zone. ${ }^{15}$ However, these findings were greater than the report of a study done in Addis Ababa ${ }^{10}$ with the availability of IPLS materials was $92 \%$. Except for the stock recording card, the availabilities of all IPLS materials in Injibara General Hospital were $100 \%$, and these results were similar to the report of the national IPLS survey conducted in PFSA. ${ }^{7}$ And it was similar to a report in the Wollega zone where the availability of bin-card and RRF was $100 \%$ but IFRR availability was $83.4 \% .{ }^{1}$ Among the IPLS materials available in both hospitals, only IFRR and RRF were prepared by the hospitals, whereas the others were donated via EPSA. The above findings reported in this study indicate the good implementation of IPLS in both hospitals. The reason for the good IPLS materials availability, maybe because most of the materials were donated by EPSA.
Among the 10 dispensing units assessed in Chagni Primary Hospital, the majority of them, $90 \%$, were utilizing bin-cards for recording the transactions of pharmaceuticals in their unit. However, only $50 \%$ of the dispensing units were updating their bin-cards regularly and this may be due to the large number of products managed in the pharmaceutical store, where updating the bin-cards for all products is time-consuming and increases the workload. The bin-card utilization reported in this study was consistent with a national IPLS survey in PFSA, but the reported percentage of updated bin-cards in Chagni Primary Hospital was poorer than the national survey. ${ }^{7}$ Conversely, it was much greater than the reports of a study done in Wollega where bin-card utilization was $33.5 \%{ }^{10}$ Besides, this study also reported that all dispensing units assessed in Injibara General Hospital were utilizing bin-cards for recording pharmaceutical transactions that happened in their units during the last 2 months and $80 \%$ of the dispensing units were regularly updating their bin-cards.

Among the IFRRs reviewed in Chagni Primary Hospital and Injibara General Hospital, $80 \%$ and $75 \%$ of the IFRRs reported valid data, respectively. From all the data reported by IFRRs, the most frequently reported invalid data in both hospitals was ending balance. This finding of the Chagni primary hospital is consistent with a study done in Dire Dawa. ${ }^{16}$ However, the report from Injibara general hospital is better than the report from Dire Dawa. ${ }^{16}$ Regarding the calculation accuracy of IFRRs, $85 \%$ and $90 \%$ of the IFRRs evaluated in Chagni Primary Hospital and Injibara General Hospital were accurate, respectively. These reports were much better than the report from the east Wollega zone, where $69.6 \%$ accurately reported. ${ }^{17}$ The discrepancy possibly because of the sample size difference utilized in both studies. The most frequently inaccurate calculation in Chagni Primary Hospital was the quantity need to reach maximum, whereas the most frequently inaccurate calculation in Injibara General Hospital was the maximum stock quantity.

The study discovered that all RRFs reported from both Chagni Primary Hospital and Injibara General Hospital were submitted on time to EPSA. These findings are better than the other reports in Ethiopia $\left(90.6 \%,{ }^{7} 85.71 \%,{ }^{15}\right.$ and 92.6\% ${ }^{10}$ Besides, all calculations of the RRFs evaluated in both hospitals were $100 \%$ accurate. The present reports are relatively much better than other studies done in Ethiopia $\left(64 \%,{ }^{10} 55.7 \%,{ }^{16}\right.$ and $\left.68.1 \%\right){ }^{7}$ Among all RRFs assessed in Chagni Primary Hospital, only 66.67\% of them reported valid data and it is better than a study 
done in Addis Ababa, where 57.3\% were reported. ${ }^{10}$ From all data reported by RRFs, ending balance was the most frequently reported invalid data because it was valid only in $66.6 \%$ of the RRFs reviewed in Chagni Primary Hospital. Similarly, ending balance was the most frequently reported data in another study too. ${ }^{10}$ Besides, only $50 \%$ of the RRFs reviewed in Injibara General Hospital reported valid data and it is lower than a report from Addis Ababa. ${ }^{10}$ Among all data reported by RRFs, days out of stock was the most frequently reported data in Injibara General Hospital.

Regarding the storage conditions of the pharmaceutical store in Chagni Primary Hospital, $85.71 \%$ of the storage conditions were complying with the recommended guideline of the IPLS. According to the IPLS, at least $80 \%$ of the storage conditions should meet the standards; therefore, the status of storage conditions in this hospital is consistent with the recommended standard. This report is slightly better than the findings of the studies from the Jimma and Wollega zones, where $70.6 \%{ }^{15}$ and $79 \%{ }^{1}$ were reported, respectively. Additionally, $71.43 \%$ of the storage conditions of the pharmaceutical store in Injibara general hospital met the standard storage guidelines recommended with IPLS and this finding is consistent with a study done in the Jimma zone. ${ }^{15}$ The status of storage conditions in this hospital is below the standard set by IPLS, which is $80 \%$. It may be due to the workload on the store managers which was reported as a challenge for IPLS implementation and which might also be augmented by the emergency of COVID- 19 .

\section{Conclusion}

The study provided important insight into the status of IPLS implementation at Chagni Primary Hospital and the Injibara General Hospital. Despite the COVID-19 pandemic effect was so high in the country, the status of most of the IPLS implementation indicators in both hospitals was encouraging. Especially, the availabilities of IPLS materials and the calculation accuracy of both RRFs and IFRRs were encouraging in both hospitals. However, the validity of the data reported in IFRRs and RRFs and the status of the storage conditions in both hospitals need some improvement. Therefore, managers in both hospitals should encourage the staff to improve the validity of the logistics data. Furthermore, because the majority of the staff responsible for IPLS implementation were not trained, managers should communicate with other responsible stakeholders and provide training.

\section{Limitation of the Study}

Because this study only examined the status of IPLS implementation in two hospitals, the findings cannot be generalized to other health facilities. Furthermore, only some of the IPLS operations were examined in this study; however, it would be preferable if all IPLS activities were examined. Therefore, the investigators recommend that future researchers in this area should consider these limitations in their work.

\section{Data Sharing Statement}

The datasets generated during and/or analyzed during the current study are available from the corresponding author on reasonable request.

\section{Acknowledgments}

We are thankful to the staff of Chagni Primary Hospital and Injibara General Hospital for their cooperation and support in providing the requisite data and willingness to provide information through interviews.

\section{Author Contributions}

All authors made a significant contribution to the work reported, whether that is in the conception, study design, execution, acquisition of data, analysis and interpretation, or in all these areas; took part in drafting, revising or critically reviewing the article; gave final approval of the version to be published; have agreed on the journal to which the article has been submitted; and agree to be accountable for all aspects of the work.

\section{Funding}

There was no funder for this study.

\section{Disclosure}

The authors reported no conflicts of interest for this work.

\section{References}

1. Alemu T, Jemal A, Gashe F, Suleman S, Sudhakar S, Fekadu G. Integrated pharmaceutical logistics system implementation in selected health facilities of Ethiopia: the case of four wollega zones. Res Soc Adm Pharm. 2020.

2. World Health Organization. Equitable Access to Medicines for Universal Health Coverage by 2030. World Health Organization; 2017.

3. Goodarzian F, Taleizadeh AA, Ghasemi P, Abraham A. An integrated sustainable medical supply chain network during COVID-19. Eng Appl Artif Intell. 2021;100:104188. doi:10.1016/j.engapp ai.2021.104188 
4. Farquharson E, Torres de Mästle C, Yescombe ER, Farquharson E, Torres de Mästle C, Yescombe ER. Managing Drug Supply: The Selection, Procurement, Distribution, and Use of Pharmaceuticals. second ed. Arlington: Kumarian press; 2011:111-131.

5. Fariba Goodarzian H-N, Jesús Muñuzuri M-BF, Muñuzuri J, Fakhrzad M-B. A multi-objective pharmaceutical supply chain network based on a robust fuzzy model: a comparison of meta-heuristics. Appl Soft Comput. 2020;92:106331. doi:10.1016/j. asoc. 2020.106331

6. Zahiri B, Jula RT-M, Tavakkoli-Moghaddam R. Design of a pharmaceutical supply chain network under uncertainty considering perishability and substitutability of products. Info Sci. 2018;423:257-283. doi:10.1016/j.ins.2017.09.046

7. Shewarega A, Dowling P, Necho W, Tewfik S, Yiegezu Y. Ethiopia: National Survey of the Integrated Pharmaceutical Logistics System. AugustAIDSFree, Pharm Supply Agency; 2019:84.

8. Gazeta FN. Drug fund and Pharmaceuticals Supply Agency Establishment Proclamation. 2007. 3939.

9. Standard Operating Procedures (SOP). Manual for the Integrated Pharmaceuticals Logistics System in Health Facilities of Ethiopia. 2nd ed. Pharmaceuticals Fund and Supply Agency; 2015:124.

10. Tilahun A, Geleta DA, Abeshu MA. Assessment of integrated pharmaceutical logistic system for the management HIV/AIDS and tuberculosis laboratory diagnostic commodities in public health facilities in Addis Ababa, Ethiopia. J Pharma Care Health Sys. 2016;3 (2): $1-10$.
11. Project UD. Monitoring and Evaluation Indicators for Assessing Logistics Systems Performance. 2006. 48.

12. Mihret H, Atnafu A, Gebremedhin T, Dellie E. Reducing disrespect and abuse of women during antenatal care and delivery services at injibara general hospital, Northwest Ethiopia: a pre-post interventional study. Int $J$ Womens Health. 2020;12:835-847. doi:10.2147/ IJWH.S273468

13. USAID | DELIVER PROJECT. Task order 1. Logistics Indicators Assessment Tool (LIAT). Arlington, USAID | DELIVER PROJECT. 2008.

14. FDRE. National Research Ethics Review Guideline. FDRE Ministry of Science and Technology. Fifth ed. Addis Ababa, Ethiopia: FDRE; 2014:95.

15. Befekadu A, Cheneke W, Kebebe D, Gudeta T. Inventory management performance for laboratory commodities in public hospitals of Jimma zone, Southwest Ethiopia. J Pharm Policy Pract. 2020;13 (1):1-12. doi:10.1186/s40545-020-00251-1

16. Tola FB, Anbessa GT, Yikna BB. Anti-tuberculosis commodities management performance and factors affecting it at public health facilities in Dire Dawa city administration, Ethiopia. J Multidiscip Healthc. 2020;13:1677-1691. doi:10.2147/JMDH.S280253

17. Tiye K, Gudeta T. Logistics management information system performance for program drugs in public health facilities of East Wollega Zone, Oromia regional state, Ethiopia. BMC Med Inform Decis Mak. 2018;18(1):1-13. doi:10.1186/s12911-018-0720-9
Journal of Multidisciplinary Healthcare

\section{Publish your work in this journal}

The Journal of Multidisciplinary Healthcare is an international, peerreviewed open-access journal that aims to represent and publish research in healthcare areas delivered by practitioners of different disciplines. This includes studies and reviews conducted by multidisciplinary teams as well as research which evaluates the results or conduct of such teams or healthcare processes in general. The journal

\section{Dovepress}

covers a very wide range of areas and welcomes submissions from practitioners at all levels, from all over the world. The manuscript management system is completely online and includes a very quick and fair peer-review system. Visit http://www.dovepress.com/testimonials. php to read real quotes from published authors. 